\title{
Die Übersetzung des Historischen Lexikons der Schweiz Ein Unternehmen von sprachpolitischer Bedeutung
}

\author{
Marianne Derron Corbellari (Neuchâtel)
}

\begin{abstract}
The article deals with the translation of the Swiss Historical Encyclopaedia and is mainly based on the experiences of three collaborators.

The Encyclopaedia is being published simultaneously in the three official languages German, French and Italian as well as in an abbreviated version in Rhaeto-Romance. The multilingual work concept implies considerable administrative planning and reveals terminological obstacles, as the different regions of Switzerland have been exposed to variable historical influences.

The article describes detailed and concrete problems of translating texts on historical topics the difficulties of which are generally underestimated. The translators need not only a solid linguistic knowledge, but also of Swiss and European history from the prehistoric period to present times. Comparing the different versions of the same article is an excellent quality control of the text's linguistic and scientific level.

The fact that the Encyclopaedia is written in the national languages is to strengthen the Swiss consciousness and identity in times where English seems to become the standard communication language. The translators work with traditional methods (including Internet!), but without referring to translation programmes.
\end{abstract}

\section{$1 \quad$ Einführung}

Vor wenigen Jahren noch wurden die Studierenden der Übersetzungswissenschaft ab und zu belächelt. Die Berufswahl sei verfehlt, hiess es, denn dieses Handwerk sei zum Aussterben verdammt, bald werde man es nicht mehr brauchen. Seither hat die Informatik immense Fortschritte erzielt, und dennoch ist kein Programm verfügbar, das einen Fachtext einigermassen vernünftig übersetzen könnte. Übersetzungen, die mittels auf dem Netz zugänglichen Programmen hergestellt werden, erzeugen im Allgemeinen viel mehr Heiterkeit als Bewunderung, ja sie sind oftmals schlicht unbrauchbar.

Die "Gefahr" des technischen Fortschrittes scheint also zumindest für den Moment gebannt, so dass die Übersetzer aufatmen können: Ihre Berufsgattung scheint in dieser Hinsicht vorerst gesichert. Seit einiger Zeit jedoch zeichnet sich eine weitere Bedrohung ab, die seltsamerweise von einer Sprache selbst ausgeht, von der zunehmenden Dominanz des Englischen in allen 
Lebensbereichen. Da professionelle, gewissenhafte Übersetzer nie in eine Sprache übersetzen, die sie nicht perfekt beherrschen, könnten in Zukunft nur diejenigen gefragt sein, die Englisch als Mutter- oder Kultursprache sprechen und schreiben. Übersetzer anderer Sprachkombinationen würden so immer seltener zum Zuge kommen.

Harte Zeiten für Übersetzer? - Ich möchte im Folgenden auf ein Unternehmen eingehen, das weder von der Englischwelle erfasst ist noch mit maschinellen Übersetzungsprogrammen arbeitet, sondern der herkömmlichen Übersetzung in die Schweizer Landessprachen grösste Bedeutung beimisst. Es handelt sich um die Ausgabe des Historischen Lexikons der Schweiz (HLS) in zwölf Bänden, das in allen drei Amtssprachen der Schweiz, in einer Kurzversion sogar auf Rätoromanisch erscheinen soll. Die Enzyklopädie entsteht unter der Ägide der Schweizerischen Akademie für Geisteswissenschaften und der Schweizerischen Gesellschaft für Geschichte. Sie wird von einem Stiftungsrat beaufsichtigt und von der Eidgenossenschaft finanziert. ${ }^{1}$

Nicht Hypothesen über mögliche technische Entwicklungen im Bereich Übersetzung und über den Einfluss des Englischen sollen im Zentrum dieses Beitrages stehen, sondern eine konkrete Arbeit, die schon lange weit über den Projektzustand hinaus gelangt ist und ein nicht zu unterschätzendes sprachpolitisches Element von nationaler Bedeutung enthält. Durch die geplante Viersprachigkeit des Lexikons wird das Unternehmen zweifellos besonders aufwändig, zumal Fachgebiete betroffen sind, die im Rahmen von Übersetzungen bisher wenig beachtet wurden, obwohl sie sich durch eine sehr komplizierte und variable Terminologie auszeichnen. Denn "Geschichte" bedeutet schon lange nicht mehr Ereignis-, vor allem Militärgeschichte einzelner Staaten und Herrscher, sondern interessiert sich für alle Menschen in einem gegebenen Lebensraum, der von bestimmten wirtschaftlichen, kulturellen und sozialen Bedingungen geprägt ist. Die heutige Geschichtswissenschaft wendet sich damit verschiedensten Disziplinen zu, die alle spezifische Kenntnisse erfordern und von ihren eigenen Methoden und ihrem besonderen Vokabular geprägt sind.

Übersetzungsverfahren wissenschaftlich zu beschreiben, ist ein beinah unmögliches Unterfangen. Es gibt keine spezifischen Methoden, nach denen der Übersetzer verfährt, es sei denn, man nenne "Methoden" die grundlegenden Gebote, dass der Übersetzer das Zielpublikum seines Textes und die terminologische Präzision beachten müsse. Es gibt höchstens Äusserungen zu einer Art Übersetzungsästhetik, also zum Ideal des Textes, das man nach der Übertragung erreichen will. Wie der Übersetzer aber im Einzelnen vorgeht, bleibt ihm selbst überlassen. Die folgenden Überlegungen erheben deshalb keinen wissenschaftlichen Anspruch, sondern verstehen sich als Fallbericht, als Beschreibung konkreter Probleme, die beim Übersetzen eines umfangreichen Werkes geisteswissenschaftlicher Richtung entstehen. In einem ersten Teil sei die Organisation der HLS-Übersetzung kurz referiert, danach folgen Fallbeispiele aus der Übersetzungspraxis; am Schluss stehen Überlegungen zur Sprachenwahl des Lexikons. Ich stütze mich dabei im Wesentlichen auf ein ausführliches Gespräch mit den HLS-Redaktoren Pierre Martin und Chiara Orelli sowie mit meinem Übersetzer-Kollegen Elmar Meier, denen ich an dieser Stelle herzlich danken möchte.

\footnotetext{
${ }^{1}$ Für allgemeine Informationen vgl. die Internet-Seite unter www.hls.ch.
} 


\section{Die Mehrsprachigkeit als administrative Herausforderung}

1988 begannen die Redaktoren des HLS, die Liste der Artikeltitel zu erstellen und gaben 1991 erste Artikel in Auftrag; nur ein Jahr später setzten bereits die Übersetzungen ein. Die Übersetzungstätigkeit ist für die italienische Redaktion am intensivsten, da diese lediglich 5-7\% der Original-Artikel herausgibt. 70\% aller Originale werden auf Deutsch verfasst, die restlichen Artikel in französischer Sprache. Aus politischen Gründen soll jeder Band des HLS in allen Amtssprachen gleichzeitig erscheinen; die ersten drei Bände liegen inzwischen auf Deutsch, Französisch und Italienisch vor. So erforderte die Mehrsprachigkeit des Lexikons von Anfang an ein hohes Mass an Planung, das grösstenteils den Zwängen des Alphabets unterliegt; dazu ein paar Beispiele:

Das Lemma 'Zeughaus' wird erst im letzten Band der deutschen Ausgabe stehen, die französische Übersetzung aber lautet 'Arsenaux', die italienische 'Arsenali'. Da der Artikel 'Zeughaus' original deutsch ist, musste er rasch ins Französische und Italienische übersetzt werden, damit er für den ersten Band dieser Sprachen bereit war. Der Artikel über die Habsburger hingegen erscheint zwar im ersten Band der italienischen Edition, weil das entsprechende Lemma 'Asburgo' lautet, aber voraussichtlich erst im fünften der deutschen und französischen Ausgabe. Der Artikel 'Assistance' ist original französisch, italienisch lautet das Lemma 'Assistenza pubblica', deutsch jedoch 'Fürsorge'. Hier eilt zwar die Verfassung des Originals und die Übersetzung ins Italienische, doch nicht diejenige ins Deutsche. Beispiele dieser Art liessen sich beliebig erweitern. Die Folge ist, dass wegen der unterschiedlichen Alphabetisierung der Inhalt einer selben Bandnummer nicht absolut identisch ist. Die sechs Artikel über Basel sind in der französischen Ausgabe vereint im ersten Band, in der italienischen erst im zweiten. In der deutschen Edition stehen je drei Artikel zu Basel im ersten und im zweiten Band.

Trotz der intensiven Planung geriet die Übersetzungstätigkeit 1996 in Verzug. Ein Grund war die Mittelknappheit, ein anderer rührte daher, dass man die Zeit z.T. gründlich unterschätzt hatte, die es zur Erstellung einer festen Terminologie brauchte. Es zeigte sich mit aller Deutlichkeit, dass auch die Geisteswissenschaften, die im Gegensatz zu den exakten schon als "weiche" Wissenschaften bezeichnet worden sind, mit einem ganz spezifischen Wortschatz arbeiten, der punkto Genauigkeit ebenso hohe Anforderungen stellt wie naturwissenschaftliche Texte. Da dieses Fachvokabular überdies kultur-, raum- und zeitabhängig ist, stecken die Texte voller Tücken für den Übersetzer. Zudem war man anfangs der irrigen Annahme, die Prüfung der Übersetzungen werde deutlich weniger Zeit beanspruchen als die Bearbeitung der Originale. Tatsächlich ist dies ein klassischer Irrtum bei der Planung von Übersetzungen, denn die zweite und dritte Arbeitsphase werden oftmals unterschätzt: Ist der Text einmal ganz übersetzt, benötigt man in der Regel nochmals die Hälfte der bereits investierten Zeit für die Prüfung und Korrektur. Dem anfänglichen Personalmangel im HLS wurde Abhilfe geschaffen, so dass die Verspätung nun behoben ist. 


\section{$3 \quad$ Anstellung der Übersetzer}

Da das HLS nicht mit fest angestellten Übersetzern arbeitet, gibt es in den einzelnen Redaktionen keine entsprechende Abteilung. Zwar haben Übersetzungsbüros ihre Mitarbeit angeboten, doch kam die Zusammenarbeit nicht zustande, weil ihre Leistungen unbefriedigend waren. Einige Übersetzer sind aber schon seit Jahren am HLS beteiligt, so dass sie quasi als fest gelten können. Das Personal erneuert sich nach und nach, indem Abgänge laufend ersetzt werden. Z.Z. erscheinen auf der Internet-Seite des HLS 122 Übersetzer, in der Praxis arbeiten aber für die deutsche und französische Redaktion nur je ein Dutzend, für die italienische etwa 15. Die Arbeit geschieht per Mandat, wobei das Lexikon keine regelmässigen, fixen Aufträge garantieren kann.

Die beschäftigten Übersetzer haben sich spontan aus Interesse am Fach Geschichte beim HLS gemeldet. Für die Redaktion zählt weniger die eigentliche Ausbildung zum Übersetzer als die konkrete Qualität der Texte. Erstes Kriterium ist die Beherrschung der Zielsprache, zweites erst die Kenntnis des Faches und dessen Vokabulars. Wie man sich diese erworben hat - im universitären oder im Selbststudium - ist zweitrangig, von Nutzen sind Kompetenzen in der Geschichtswissenschaft aber allemal. Besondere Schwierigkeiten birgt der Fachwortschatz vor 1798, an dem schon manche Interessenten gescheitert sind. Deswegen absolviert jeder Anwärter zuerst eine Test-Übersetzung verschiedener Artikeltypen, bevor er definitiv in den Pool der Übersetzer aufgenommen wird. Stilfragen werden in einer ersten Phase etwas weniger gewichtet, trotzdem sind sie nicht einfach nebensächlich. Schliesslich lässt sich eine stilistisch mangelhafte Übersetzung nur mit grossem Aufwand bereinigen, doch ist der Stil zweifellos das subjektivste Element des Textes und lässt sich deshalb am schwierigsten qualitativ definieren. Mit dem Verfahren der Spontanauslese seiner Übersetzer hat das HLS bisher ein Maximum an Qualität erreicht.

Das freie Mandatssystem erlaubt der HLS-Administration ein hohes Mass an Flexibilität bei der Vergabe von Übersetzungsarbeiten, die ja wegen des gleichzeitigen Erscheinens der Bände in drei Sprachen unabdingbar ist. Gerade wenn finanzielle Engpässen auftreten, sind freie Mitarbeiter natürlich geeigneter als fest angestellte, die zwar den vollen Lohn beziehen würden, aber nicht immer ausgelastet wären.

\section{$4 \quad$ Beispiele aus der Übersetzungspraxis}

Die folgenden Beispiele und Überlegungen stützen sich auf die Erfahrungen zweier HLSÜbersetzer, die beide Übersetzung und Geschichte studiert haben. Auch wenn die Ausführungen in erster Linie persönlichen Charakter haben, so sind die Herausforderungen, mit denen die Übersetzer in ihrer Arbeit konfrontiert werden, gewiss für diese Art von Texten allgemein gültig.

Elmar Meier ist bereits seit 10 Jahren an der Übersetzung des HLS beteiligt. Er gelangte auf die typische informelle Art zu seinem Auftragsverhältnis, nämlich über Vermittlung eines Kollegen, der vorher selbst für das HLS übersetzt hatte und diese aus seiner Sicht übermässig aufwändige und unrentable Tätigkeit gerne an jemand anderen abtrat. Pro Jahr sind es nun etwa drei oder vier Mandate, für die er insgesamt zwei bis drei Wochen einsetzt. Marianne 
Derron Corbellari ist erst seit 2002 als Übersetzerin fürs HLS tätig. Sie meldete sich spontan beim Lexikon, das ihr eine Serie Texte zu übersetzen gab und sie darauf mit weiteren Mandaten betreute. Auch sie hat bisher drei bis vier Aufträge jährlich erhalten, könnte aber den Zeitaufwand nicht beziffern. Beide übersetzen für das HLS aus dem Französischen ins Deutsche, und nach ihrer Einschätzung ist die Arbeit finanziell durchaus interessant.

An der Arbeit besonders reizvoll finden sie den hohen Lernfaktor, der eine ganz spezielle Motivation darstellt. Die Lexikonartikel sind eine hervorragende Gelegenheit, das selbst erworbene Fachwissen à jour zu halten und zu erweitern. Wichtig ist ebenfalls der Aspekt des Networkings: Dank der Übersetzung gelangt man zu Spezialisten und Werktiteln, deren Kenntnis für andere wissenschaftliche Tätigkeiten von Vorteil sein könnte. Mit der Zeit entwickelt jeder Übersetzer, auch aufgrund seiner Ausbildung, Vorlieben für bestimmte Themen oder Artikeltypen, z.B. für die römische und keltische Epoche oder für Wirtschafts-, Sozial und Kulturgeschichte.

Das Übersetzungsstudium, das Marianne Derron in Genf durchlaufen hat, orientierte sich vor allem an Texten aus Bereichen, mit denen die Studierenden im späteren Berufsleben vorrangig konfrontiert wurden: Rechts-, Wirtschafts- und Werbetexte, gelegentlich auch medizinische Schriften. ${ }^{2}$ An ihnen liessen sich exaktes Vokabular und Ausrichtung auf eine bestimmte Zielgruppe bestens trainieren. Vielfach wurde dabei die Eleganz der Sprache hemmungslos dem reinen Informationswert geopfert, ja man lehrte die Studierenden sogar, Wortwiederholungen und die daraus resultierende, stilistische Schwerfälligkeit seien hinzunehmen, solange nur das Fachwort richtig sei. Dies ist umso weniger verständlich, als gerade Sprach- und Stilsensibilität den guten vom rein korrekten Übersetzer unterscheiden. Weil Fachtexte der Humanwissenschaften zugleich terminologische Präzision und stilistische Eleganz erfordern, schätzen Marianne Derron und Elmar Meier die Arbeit am HLS besonders.

Fragt man die HLS-Übersetzer nach ihrem Arbeitsschema, so lauten die Antworten sehr ähnlich: Die Texte werden zunächst gründlich durchgelesen, schwierige Stellen sogleich markiert. Darauf folgt eine erste terminologische Recherche, danach die Erstübersetzung, die erneut von der Suche nach den korrekten Äquivalenten begleitet ist. Die meisten Übersetzer lassen ihren Text dann eine Weile ruhen, bevor sie ihn wieder einer gründlichen Lektüre unterziehen. Geprüft wird der Gesamteindruck: Klingt der Text in seiner neuen Sprache flüssig, wirkt die Idiomatik echt? Auch in dieser Phase werden einzelne Termini noch präzisiert.

Fachausdrücke sind zwar lern- und recherchierbar, doch muss das Auge dafür geschult sein, denn Fallen sind immer möglich. Der Limnologe François-Alphonse Forel z.B. untersuchte den Genfersee und interessierte sich u.a. für dessen seiches. Tintenfische in Schweizer Gewässern? Nein, die Wissenschaft der Wellenbewegungen ist gemeint. Einen klassischen Fall bilden die "Tagsatzungsabschiede". Es handelt sich nicht etwa um die adieux, sondern um die recès de la diète. ${ }^{3}$ Mit Sinn für Logik, Gewissenhaftigkeit und Geschichtskenntnissen lassen

\footnotetext{
2 Pierre-G. Martin und Ursula Gaillard hatten 1996 Gelegenheit, im Centre de traduction littéraire (CTL, Faculté de lettres, Université de Lausanne) einen Workshop mit Texten des HLS durchzuführen.

${ }^{3}$ Weitere Beispiele in Martin (2001: 26).
} 
sich solche groben Schnitzer vermeiden. Mangelnde Kenntnisse der Zielsprache jedoch sind kaum zu kompensieren.

Das HLS besteht aus Einträgen zu Personen, Gemeinden und Kantonen, politischen und wirtschaftlichen Institutionen, soziokulturellen Phänomenen und geschichtlichen Ereignissen. Der Typ des Artikels und die damit verbundene Länge beeinflussen den Übersetzungskomfort entscheidend. Zahlreiche Artikel geographischer Art betreffen kleine Gemeinden, z.B. Farvagny (Original Französisch):

Comm. FR, distr. de la Sarine, créée le ler janvier 1996 par la fusion des anciennes comm. de Farvagny-le-Grand, Farvagny-le-Petit, Grenilles et Posat. 1775 hab. en 2000.

Polit. Gem. FR, Bez. Saane, entstanden am 1. Jan. 1996 durch die Fusion der ehemaligen Gem. Farvagny-le-Grand, Farvagny-le-Petit, Grenilles und Posat. 20001775 Einw.

Ganz anders präsentiert sich der Artikel über die Gemeinde der Stadt Bern, der im zweiten Band des HLS (S. 234-251) kürzlich erschienen ist. Ihn in seiner gesamten Länge zu zitieren, ist natürlich unmöglich, zumal er noch zahlreiche Graphiken enthält. Er behandelt drei grosse Zeitspannen: Urgeschichte bis Frühmittelalter (S. 235f.), Hochmittelalter bis Ende des Ancien Régime (S. 236-242) sowie 19. und 20. Jahrhundert (S. 242-251). Die ersten zwei Teile gehen auf je einen Verfasser zurück, der letzte sogar auf drei. Artikel, die zwei oder mehrere Zeitabschnitte behandeln, tragen des öfteren die Autorschaft mehrerer Personen. Der - noch nicht erschienene - Artikel zur Fürsorge z.B. ist das Werk zweiter Autoren, einer Spezialistin für die Periode Mittelalter bis 1914 und eines Experten für das 19. und 20. Jahrhundert. Zur Übersetzung gelangte der Artikel aber nicht aufgeteilt, sondern als Ganzes, wie es in der Regel üblich ist. Es wäre zu aufwändig, die Artikel auch unter mehreren Übersetzern aufzuteilen; wenngleich die Affinitäten der Übersetzer zu bestimmten Themenkreisen berücksichtigt werden, falls es möglich ist.

Von der Länge her nimmt Evolène eine Mittelstellung ein und bietet sich deshalb als anschauliches Beispiel an, wie eine Gemeinde mit relativ niedriger Einwohnerzahl zahlreiche geschichtliche Stadien durchlaufen hat, die von ihrem je eigenen Vokabular geprägt sind:

Der Raum Evolène enthält Siedlungsspuren, die bis in die Jungsteinzeit zurückreichen, u.a. pierres à cupules. Dieser Ausdruck ist zwar Archäologen bekannt, aber nicht der Übersetzerin, da sie sich nie eingehend mit Urgeschichte beschäftigt hat. Schnelle Hilfe lieferte der Konservator des Latenium-Museums von Neuenburg: "Schalenstein" heisst der deutsche Begriff. Im Passus über die mittelalterliche Geschichte der Gemeinde tauchte der Ausdruck métralie auf. Dank einem französischen Geschichtsglossar auf dem Internet fand die Übersetzerin die Definition zwar nicht der Métralie, aber immerhin des Métral: officier, chef de certaines juridictions inférieures, régisseur d'agent fiscal dans un certain ressort, perçoit les revenus du seigneur et juge en première instance les cas qui concernent cette perception. Charge souvent concédée en fief. Die Übersetzerin wählte das neutrale "Herrschaft", wies die Redaktion aber auf die Schwierigkeit hin; jene korrigierte in der Folge zu "Mistralie". Die Neuzeit Evolènes erwies sich als einfacher: pierre ollaire fand sich im allgemeinen Wörterbuch ("Lavezstein"); bei der Société de Grande Dixence genügte ein Anruf, um herauszufinden, dass im Deutschen der Name Grande Dixence $S A$ übernommen wird. 
Auf den ersten Blick mag es also leichter scheinen, Kurzartikel wie denjenigen über Farvagny zu übersetzen. Erhielte der Übersetzer wirklich einen Stapel von Einträgen über Gemeinden, die alle Farvagny glichen, wäre die Aufgabe tatsächlich eher leicht, ja sogar öde. Nun enthalten aber Kurzeinträge - seien sie geographischer oder biographischer Art - einen z.T. geballten Informationswert und sind arbeitsökonomisch undankbar, weil der Übersetzer für wenige Zeilen viel Fachwortschatz zusammentragen muss. Auf engem Raum kann sehr viel Unterschiedliches und auch Technisches zusammenstehen, so die Pflaumenplantagen und PeugeotWerke in einem jurassischen Weiler, ein Berner Kunstmaler mit Vorliebe für phantastische Sujets oder der aus dem sog. Vorort hervorgegangene Verband Economiesuisse. Der Übersetzer springt buchstäblich vom Hundertsten ins Tausendste, muss aber für jedes Fachgebiet gleich viel terminologische Sorgfalt aufwenden. So kann eine Serie Kurzartikel den Übersetzer länger beschäftigen als ein langer Artikel über ein selbes Thema, dessen Zeilenzahl insgesamt gleich ist.

Nebst dem Inhaltlich-Begrifflichen bieten kürzere Texte aber auch stilistische Nachteile, denn sie verlangen nach einem gerafften, elliptischen Stil. Man gewöhnt sich dabei eine Syntax an, die zwar für kurze Lexikoneinträge geeignet ist, aber bei jedem anderen längeren Text stört. Gemindert wird die Frustration des Übersetzers allenfalls durch die mannigfaltigen Kenntnisse, die er sich bei solchen Artikeln erwirbt. Längere Artikel hingegen erlauben es dem Übersetzer, sich thematisch zu vertiefen und den Stil zu pflegen. Die terminologische Recherche gestaltet sich einfacher, weil pro Artikel nur ein Spezialgebiet betroffen ist. So ist es angenehmer, den Eintrag über die französische Revolution oder das schweizerische Fürsorgewesen zu übersetzen, als eine Serie von Kurzbiographien.

Weitaus am schwierigsten sind aber Texte, deren Thema im Mittelalter angesiedelt ist, und dies ungeachtet ihrer Länge. Der juristisch-wirtschaftliche Rahmen dieser Epoche ist so grundlegend verschieden, dass nur ganz spezifische, heute kaum mehr gebräuchliche Termini zur Anwendung kommen. Es ist weiter zu bedenken, dass die Urkundensprache des Mittelalters im Prinzip Latein war und viele Begriffe nur unscharf in die Volkssprache übersetzt sind. Zudem bewahrte die mittelalterliche Rechts- und Wirtschaftssprache eine sehr lange Gültigkeit. Auch nach dem Jahr 1500, nach dem gemeinhin der Beginn der Neuzeit angesetzt wird, blieben feudale Strukturen bestehen; sie verschwanden erst mit dem Fall des Ancien Régime, ja Überreste bestanden noch bis ins 19. Jahrhundert hinein. Sprachlich ist das Mittelalter deshalb äusserst lange präsent und erfordert besondere Sorgfalt, Nachforschungen und natürlich auch Fachwissen. Allgemeine Wörterbücher sind nur für sehr häufige Begriffe zu gebrauchen, z.B. für évêque ("Bischof") oder château ("Schloss"). Solche Wörter braucht der Übersetzer jedoch gar nicht nachzuschlagen, da sie ja zum Grundwortschatz gehören. Wie aber steht es mit "Fideikommiss" oder "Twingherrenstreit"?

Seit Anbruch des Internet-Zeitalters ist die Suche nach dem richtigen Ausdruck entscheidend leichter geworden. Mittels Suchmaschinen auf dem Netz sind zahlreiche Informationen schnellstens verfügbar, so dass sich ein guter Teil der Sucharbeit vor dem Bildschirm abspielt. Was früher umständlich in Sachbüchern zusammengesucht wurde, ist heute meist elektronisch verfügbar, so dass viele zeitraubende Gänge in Fachbibliotheken wegfallen. Am frucht- 
barsten ist die gezielte Recherche über das E-HLS: Dank Passwort haben die Übersetzer Zugriff auf alle bisher für das Lexikon verfassten Texte und können dank Volltextsuche und Stichwortliste zahlreiche Termini erschliessen. Gibt ein Autor im Literaturverzeichnis Artikel an, die bereits in der Zielsprache stehen, so sind diese eine nützliche Stütze. Mehrsprachige Lexika des Fachbereichs Geschichte sind leider sehr selten; ${ }^{4}$ im Allgemeinen ist es ratsam, in einem Geschichtswerk der Ausgangssprache die Definition eines Begriffes nachzuschlagen und sich dann an einen Experten der Zielsprache zu wenden.

Problematisch ist es, wenn Äquivalente in der Zielsprache gar nicht bestehen und zuerst geschaffen werden müssen. Ereignisse wie die Reformation, die französische Revolution oder natürlich die Schaffung des Bundesstaates hatten Auswirkungen auf die gesamte Schweiz, so dass entsprechende Termini in allen Landessprachen vorhanden sind. Hier sind einsprachige Wörterbücher, Sammelbände zu einem bestimmten Themenbereich oder Monographien tatsächlich von Nutzen (z.B. Haberkern/Wallach 2001). Weitaus schwieriger gestaltet sich die Übersetzung, wenn gewisse Phänomene lediglich bestimmte Räume der Schweiz betreffen, z.B. regional bedingte Erwerbsformen. Da vor allem in der Ost- und Zentralschweiz die Protoindustrialisierung einsetzte, ist die auf dem sog. Verlagssystem beruhende Heimarbeit eine typische Erscheinung dieser Region. Frankophone Historiker übernehmen den Term "Verlagssystem" 5 deshalb unübersetzt in ihre Sprache. Umgekehrt ist die Uhrmacherei im Jura, in Neuenburg und in Genf beheimatet, so dass es manchmal schwierig ist, Begriffe dieses Metiers, die schon lange im Deutschen gebräuchlich sind, zu finden; sie wirken im wahrsten Sinne des Wortes "übersetzt". Ebenso war die Produktion bedruckter Baumwolle ein Erwerbszweig des frankophonen Genf, Neuenburg und Mülhausen. Die Stoffe heissen auch auf Deutsch Indiennes, nach einer aus Indien stammenden Technik. Hilfreich sind in solchen Fällen mehrsprachige Museumskataloge, z.B. des Musée de l'Horlogerie von La Chaux-deFonds oder die Kataloge des schweizerischen Landesmuseums von Zürich und Prangins.

Letztes Mittel bleibt der Hinweis an die zuständige Redaktion, dass man einen Begriff nicht gefunden hat oder sich dessen Übersetzung nicht sicher ist. Grundsätzlich gibt der Übersetzer Zweifel zu Begriffen, Überlegungen etwa zur Logik des Textes oder Zusätze, z.B. zum Literaturverzeichnis der Artikel, im Text selbst in eckigen Klammern an. Hier geht die Arbeit des Übersetzers über das rein Sprachliche entschieden hinaus.

Die Erstellung eines mehrsprachigen historischen Lexikons wäre eine hervorragende Gelegenheit, ein ebenfalls mehrsprachiges Wörterbuch für Geschichte zu schaffen. Die Grundlagen zu einem Glossar sind zwar gelegt, doch aus Zeitgründen leider nicht weitergeführt worden. ${ }^{6}$ Vielleicht ergibt sich nach Abschluss des Projektes die Möglichkeit, auf Basis des bereits Erarbeiteten weiterzufahren. Das Interesse der Übersetzer wäre jedenfalls vorhanden.

\footnotetext{
${ }^{4}$ Nützlich, aber leider sehr schlank ist das Bändchen von Drummer/Julien/Loscertales/Stammerjohann (eds.) (1991). Zweisprachige Nachschlagewerke sind das Historisch-biographische Lexikon der Schweiz (1921-1934), das Geographische Lexikon der Schweiz (1902-1910) und Die Schweiz vom Paläolithikum bis zum frühen Mittelalter (1993). Ins Französische übersetzt worden sind die Monographien Drack/Fellmann (1988) und Vischer (1998).

5 Das deutsche Original zum Verlagssystem besteht bereits, ist aber noch nicht übersetzt worden.

${ }^{6}$ Das Glossar ist intern zugänglich; erschienen ist jedoch der Teil der Flurnamen in Furrer 1991.
} 


\section{$5 \quad$ Zusammenarbeit von Übersetzern und Redaktoren}

Die Redaktoren, alle Historiker, geben die Endfassungen der Texte frei, sei es in der Autorenoder in der Übersetzungsversion. Folgende Punkte beachtet der Redaktor bei der Durchsicht einer Übersetzung: ${ }^{7}$

- Inhaltliche Entsprechung von Original und Übersetzung

- Präzision des Fachwortschatzes

- Sprachrelevanz des Inhalts

- Namen von Institutionen, Vereinen und Gesellschaften

- Formale Zwänge (Zeilenzahl, Abkürzungen, Querverweise)

- Stil des Textes

Der erste Punkt ist so wesentlich, aber auch so selbstverständlich, dass er hier keiner näheren Erläuterung bedarf. Die terminologischen Hürden sind bereits besprochen worden, deshalb nun kurz zu den anderen Punkten:

Wenn im Ausgangsartikel etymologische Erklärungen stehen, sind diese im Hinblick auf die Zielsprache anzupassen. So steht neben dem deutschen Lemma "Vogt" der Hinweis, dass der Begriff vom lateinischen advocatus stammt; das französische entsprechende Lemma lautet bailli, das freilich nicht von advocatus, sondern von balivus stammt.

Vorsicht geboten ist bei Namen von Vereinen und Gesellschaften, die je nach Wirkungsgebiet in mehreren Sprachen offizielle Bezeichnungen tragen. Dankbar ist der Redaktor laut PierreG. Martin, wenn der Übersetzer den korrekten Namen einer Gesellschaft solide recherchiert hat - mit entsprechendem Hinweis in eckigen Klammern - und nicht etwa aufs Geratewohl geschaffen hat. Damit wird der Redaktor um ein gutes Stück Arbeit entlastet, weil er nicht mehr selbst zu Internet und Telefon greifen muss. Es kommt vor - dies ein erschwerender Umstand -, dass sich offizielle Namen zwischen Abfassung des Artikels und Vergabe zur Übersetzung ändern. So ist die Banque de l'Etat de Fribourg heute nicht mehr die "Freiburger Staatsbank", sondern die "Freiburger Kantonalbank". Der Schweizerische Eisenbahner Verband ist zum Schweizerischen Eisenbahn- und Verkehrspersonal-Verband geworden, und das Magazin Cheminot nennt sich heute Evénement syndical, zu deutsch "Arbeit und Verkehr".

Was für die Übersetzung gilt, trifft ebenfalls für die Redaktion zu: Kurzeinträge erfordern nicht unbedingt weniger Zeit zur Durchsicht der Texte, weil diese oft reich an komplexen Begriffen sind. Problematisch sind z.B. Biographien von Notabeln des Ancien Régime oder Texte mit Kapiteln zur Rechts- und Territorialgeschichte des Frühmittelalters. Die Mühen des Übersetzers wiederholen sich hier auf redaktioneller Stufe.

Da das HLS das Werk zahlreicher Beteiligter ist, sind gewisse formale Zwänge zu beachten, damit die Einheit des Lexikons gesichert ist: Der Umfang des Originals darf höchstens geringfügig überschritten werden; Abkürzungen sollten immer gleich verwendet werden, die Querverweise auf andere Artikel so übersetzt, wie es das Lemma auf der Stichwortliste vorgibt. Für jeden, der bereits an einem grösseren wissenschaftlichen Werk beteiligt war, klingen

\footnotetext{
${ }^{7}$ Vgl. dazu auch Martin (2001: 25-27).
} 
solche Vorgaben selbstverständlich, ja banal. Ihre praktische Umsetzung ist aber umso schwieriger, wenn das Werk in mehreren Sprachen erscheinen soll und jeder Übersetzer und Redaktor wieder einzeln darauf achten muss. Je mehr Personen am Werk sind, desto mehr Fehlerquellen können auftreten. Es ist in erster Linie Aufgabe der Redaktoren, über die Einheitlichkeit und Kohärenz des Gesamtwerkes zu wachen und die übersetzten Texte gegebenenfalls anzupassen. Um aber die Zeit, die für derartige Formalien aufgewendet wird, in vernünftigen Grenzen zu halten, ist eine gute Absprache zwischen Übersetzern und Redaktoren unabdingbar.

Die einzelnen Arbeitsschritte der Redaktoren werden individuell geregelt. Einige ziehen es vor, sich zuerst nur in die Übersetzung zu vertiefen und zu sehen, ob sie wie ein eigenständiger Text klingt; andere beginnen mit einer synoptischen Lektüre der Texte. Dass an der Übersetzung gar nichts geändert wird, kommt praktisch nicht vor. Rücksprachen mit dem Übersetzer sind selten; allerdings könnten auf diese Weise einige Unklarheiten manchmal schneller bereinigt werden, räumt Pierre-G. Martin ein. Mit dem Autor halten die Redaktoren nur Rücksprache wegen des Originals, nicht wegen der Übersetzung. Seit man erst den bereinigten Text, also das definitive Original zum Übersetzen freigibt, sind etliche Probleme und viel mühsame Redaktionsarbeit beseitigt worden.

\section{Automatik oder Mensch: Leistungen eines Übersetzungsprogramms}

Überwältigend einheitlich fällt die Reaktion aus, wenn man Übersetzer und Redaktoren nach dem Nutzen eines Übersetzungsprogramms fragt: Jedermann begegnet der Automatik mit grösster Skepsis. Der Versuch mittels der Übersetzungshilfe von Google soll aber gewagt werden, und zwar gerade an einem Text mittelalterlicher Thematik, der für den menschlichen Übersetzer so reich an Tücken ist. Auf Vorschlag Pierre-G. Martins dient als Test die Einleitung zum original deutschen Artikel 'Bürgschaft', der bereits auf herkömmliche Weise ins Französische übertragen worden ist. Die problematischsten Stellen sind in allen drei Versionen hervorgehoben.

\section{Original:}

\section{Bürgschaft}

Die B. - eine uralte Institution (1. Mose 43,9) - war unter vielerlei Formen bekannt: Die spä-

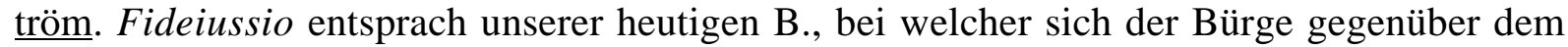
Gläubiger des Hauptschuldners verpflichtet, für die Erfüllung der Schuld einzustehen. Dagegen bestand die B. der Germanischen Stammesrechte darin, dass der Schuldner dem Gläubiger einen Bürgen stellte, der nun primär haftete, und zwar mit seinem Leib, nicht mit seinem Vermögen. Um der Gefangennahme durch den Gläubiger zu entgehen, setzte der Bürge alles daran, den Hauptschuldner zur Pflichterfüllung zu zwingen (vgl. auch Geiselschaft).

Die aus den Stammesrechten bekannte Schuldknechtschaft war die strengste Form der Leibhaftung; neben gesetzl. Haftung in der Sippe verlangte auch das spätma. Herrschaftsrecht von Genossen und Stadtbürgern die B. ("hand und pfand sein") für Schulden der Herrschaft. Stadtrechte liessen seit dem 13. Jh. die eigenmächtige Menschenpfändung nicht mehr zu. Bundesbriefe und Einzelverträge richteten sich gegen die Leibhaft für Herren (z.B. Burgdorf gegen die Gf. von Kyburg 1331); später befreiten sich auch ländl. Genossenschaften von der drükkenden Pflicht (z.B. Niedersimmental 1393-98). 


\section{Übersetzung durch Google:}

\section{Garantie}

Les b. - une institution historique (1er Mose 43,9) - sous différentes formes: spaetroem a eu connaissance celui. * fideiussio correspondre notre actuel B., avec qui lui-même le garant par rapport le créancier débiteur obliger, le réaliser le dette répondre. En revanche il a réussi les $B$. des droits de tronc germaniques dans que le débiteur n'a pas placé au créancier un garant qui adhérait maintenant d'abord, et ce, avec son corps, avec sa fortune. Pour aller au-devant à la capture par le créancier forcer a placé le garant tout, les débiteurs principaux à la réalisation de devoir (cf. aussi).

Le Schuldknechtschaft connu des droits de tronc était la forme la plus stricte de responsabilité de corps; à côté gesetzl. La responsabilité dans la parenté a exigé aussi cela spaetma. Droit de pouvoir des camarades et des citoyens urbains les B. ("détention et caution son") pour les dettes du pouvoir. n'ont plus permis la saisie humaine eigenmaechtige depuis le 13 ème Jh.. Des lettres fédérales et des contrats particuliers se sont adressés contre la détention de corps pour des messieurs (p. ex. village de château contre les AP du Kyburg 1331); on se libère plus tard aussi laendl. Coopératives du devoir lourd (p. ex. Niedersimmental 139398).

\section{Übersetzung durch den Menschen:}

\section{Cautionnement}

Institution immémoriale (cf. Genèse 43,9), la cautionnement connut bien des formes: la 'fideiussio' du Bas-Empire romain correspondait à la pratique actuelle, où la caution s'engage envers le créancier à garantir le règlement de la dette du débiteur principal. En revanche, dans les lois barbares, il s'agissait pour le débiteur de mettre à la disposition du créancier un répondant (la caution) qui se portait premier garant, mais avec son corps et non pas avec ses biens. Pour éviter la prise de corps par le créancier, la caution s'efforçait d'obliger le débiteur principal à s'acquitter de son obligation (voir aussi Otages).

L'esclavage pour dettes, connu des codes barbares, était la forme la plus rigoureuse de la contrainte par corps; le droit féodal du bas Moyen Age exigeait qu'outre la famille du seigneur, des communiers et des bourgeois cautionnent les dettes de la seigneurie. Dès le XIIIe s., des droits municipaux, des pactes et des contrats individuels rejetèrent ces pratiques et les saisies de corps arbitraires (Berthoud par exemple s'en defend contre les comtes de Kibourg en 1331): Par la suite, des communautés rurales s'en libérèrent aussi (par exemple le Niedersimmental en 1393-1398).

Der von Google übersetzte Text bedarf keiner langen Erläuterungen: Man erkennt gerade noch, worum es im Artikel geht, ansonsten ist die Übertragung in dieser Form unbrauchbar. Über folgende Hürden ist das Programm gestolpert:

1. Seltene Wörter wie "eigenmächtig" oder "Schuldknechtschaft" übersetzt es nicht, da es sie nicht kennt.

2. Mit den Abkürzungen - "spätröm., spätma., gesetzl. Gf., Jh." - bekundet es Mühe. Besonders schwierig sind die ersten zwei, die Komposita darstellen. Tardif und romain bzw. médiéval lassen sich nicht einfach aneinander reihen, sondern müssen in den Wendungen $d u$ 
Bas-Empire romain bzw. du bas Moyen Age übersetzt werden. Syntaktisch sind diese freilich schwieriger in den Text einzugliedern als ein einzelnes Wort.

3. Das Programm glaubte vielerorts, die eigentlichen Fachbegriffe zu erkennen, meist aber wenig erfolgreich. Das sprechendste Beispiel sind sicher die droits de tronc germaniques für die "germanischen Stammesrechte".

4. In Syntax und Grammatik herrscht ein Durcheinander, gerade die Verben bleiben oft unübersetzt, der Singular wird nicht immer vom Plural unterschieden. So wird der "Hauptschuldner", Singular im deutschen Text, zu débiteurs principaux in der Übersetzung.

5. Einen interessanten Fall bildet die "uralte Institution": Hier entscheidet eben der Kontext, dass es sich nicht um eine institution historique, sondern um eine institution immémoriale handelt.

Die Fachbegriffe und die Abkürzungen sind zweifellos noch das geringste Problem, denn sie können einprogrammiert werden. Allerdings ändern sich die Fachbegriffe von einer Epoche zur andern; das Programm müsste also an einem Element des Textes erkennen können, um welchen Zeitabschnitt es sich handelt. ${ }^{8}$ Wahrhaft problematisch sind die Punkte 4 und 5. Es ist offensichtlich, dass die Zeit, die man mittels Programm bei der reinen Übersetzung allenfalls gewinnt, in die Überarbeitung und Bereinigung des Maschinentextes reinvestiert werden müsste. Die Arbeit würde sich also nicht verringern, sondern höchstens verschieben. Als allgemeine Zurückweisung jeglicher informatischer Übersetzungshilfe ist dies aber nicht zu verstehen. Es gilt auch hier, den neusten Entwicklungen in diesem Bereich offen gegenüber zu stehen und sie anzuwenden, sofern sie sinnvoll sind. Der eben angestellte kurze Vergleich soll - obwohl krass im Resultat - nicht etwa eine pauschale Verurteilung von Übersetzungsprogrammen sein, aber er zeigt deutlich, wie schwierig es ist, ein befriedigendes Programm zu entwickeln. Es ist höchst unrentabel, wenn die Korrekturarbeit an Übersetzungen ins Unermessliche wächst, weil Maschinen anstelle von Menschen am Werk sind. Ein Übersetzungsprogramm kann dem Menschen (vielleicht!) helfen, ihn aber nicht ersetzen.

\section{$7 \quad$ Das HLS als Produkt des Föderalismus}

Es ist unbestreitbar, dass die Übersetzung des HLS nicht nur auf dem Bestreben fusst, jeder Schweizerin und jedem Schweizer der reinen Benutzerfreundlichkeit wegen ein Lexikon in der Muttersprache zu liefern, sondern ebenso von (sprach)politischen Fragen motiviert ist. Wenn nämlich der Ausdruck "Sonderfall" für die Schweiz zutrifft, dann gewiss auf deren Sprachenvielfalt. Zwar war die Mehrsprachigkeit des Gesamtwerkes von Beginn weg (1987) vorgesehen, trotzdem gingen dem Entscheid, die vier Landessprachen der Schweiz zu berücksichtigen, mannigfaltige Überlegungen und Diskussionen voraus.

Da der Vorgänger des HLS, das Historisch-biographische Lexikon der Schweiz, ebenfalls deutsch und französisch erschienen war, ${ }^{9}$ löste das Konzept einer zweisprachigen Ausgabe

\footnotetext{
${ }^{8}$ Man vgl. dazu die Beispiele Amt, Oberamt, Vogtei, Bezirk, Distrikt, die Martin (2001: 4) in seinem Artikel anführt.

${ }^{9} \mathrm{Vgl}$. Anm. 4.
} 
des HLS keine Einwände aus. Hingegen stellte sich die Frage nach dem Nutzen anderer Sprachen: Wäre eine englische Version opportun, da sich doch Englisch weltweit immer stärker als Kommunikationssprache durchsetzt? Ist eine italienische Ausgabe ein Luxus oder eine Notwendigkeit? Welchen Raum will man dem Rätoromanischen zugestehen?

Dass auch das Italienische eine vollständige Lexikon-Ausgabe erhalten soll, hängt nicht zuletzt vom Umstand ab, dass zur Zeit der Planung ein Tessiner Bundesrat, Flavio Cotti, das Eidgenössische Departement des Inneren (EDI) leitete. Er hat dazu beigetragen, dass die föderalistische Struktur der Schweiz, die Pflege der sprachlichen Minderheiten und eine Kulturpolitik, die den verschiedenen Landesteilen in gleichem Masse Rechnung trug, auf die Konzeption des HLS eingewirkt haben. Eine rein deutsch-französische Ausgabe des Lexikons wäre ein Affront sogar gegenüber denjenigen Tessinern, die Deutsch problemlos lesen, da sie ja als Steuerzahler das Produkt mitfinanzieren.

Angesichts der buchstäblich verschwindend kleinen Zahl von Schweizerinnen und Schweizern rätoromanischer Muttersprache drängten sich freilich bei der vierten Landessprache Einschränkungen auf. Tatsächlich wäre eine vollständige Ausgabe in zwölf Bänden schwer zu rechtfertigen gewesen, und so beschränkt sich das Lexikon istoric retic auf einen einzigen Band, der nur die Artikel enthalten wird, welche speziell die Bündner Geschichte betreffen. Viele mögen jedoch sogar diese Kurzversion in Rätoromanisch für einen reinen symbolischen Akt halten. Wer "rumantsch" spricht, ist heutzutage zwangsläufig zweisprachig und könnte das HLS ebenso gut in einer anderen Landessprache konsultieren. Gerade in Zeiten des Sparzwangs mag man diese Ausgabe auf den ersten Blick überflüssig finden; auf den zweiten Blick aber erfüllt sie eine andere Aufgabe, die mit politischer Korrektheit nichts zu tun hat: Eine Sprache muss gepflegt werden, will man sie am Leben erhalten. Sprachpflege heisst u.a., dass man den Wortschatz in den Wandel der Gesellschaft und der Lebensbedingungen mit einbezieht. Die Übersetzung von Texten mit ganz spezifischem Vokabular löst einen sprachschöpferischen Prozess aus. Die rätoromanische Edition ist also nicht nur ein politisches Trostpflästerchen für eine Minderheit, die um ihr sprachliches Überleben kämpft, sondern ein aktiver Beitrag zu deren Kultur. Ähnliches gilt natürlich, wenn auch weniger akut, für die italienische Version.

1994/95 gingen die Subventionen wegen finanzieller Probleme um rund 10\% zurück, so dass der anfängliche Vorsatz der Mehrsprachigkeit plötzlich gefährdet schien. Die Frage, ob wirklich das gesamte Werk in allen drei Sprachen erscheinen müsse, schien unvermeidbar. Fast wäre die Übersetzung zum ersten Sparopfer geworden, doch damit nicht genug, einige zweifelten gar am Sinn des Werkes selbst: Nach Ansicht gewisser Historiker war ein Lexikon des Typs HLS nicht vorrangig, da es der reinen Vulgarisierung der Wissenschaft diene, diese aber nicht eigentlich fördere. Die Mittel, die für das HLS und besonders für dessen Übersetzung investiert würden, seien zweckmässiger für die Forschung selbst einzusetzen. Solche Stimmen blieben aber glücklicherweise minoritär und fanden in den Medien kein Sprachrohr.

Eine reduzierte französische und italienische Ausgabe nach dem rätoromanischen Modell stand trotz Finanzproblemen nie zur Diskussion, da die politische Unterstützung immer stark genug war, sogar als 2001 - zum Glück nur vorübergehend - erneut weniger Geld für die 
Übersetzungen verfügbar war. Die Erarbeitung eines historischen Lexikons soll identitätsstiftend wirken; die volle Dreisprachigkeit des Werkes soll den eidgenössischen Zusammenhalt fördern, weil sie allen Schweizerinnen und Schweizern unabhängig von ihrer Kultursprache Zugang zum Basiswissen ihres Landes verschafft.

Obwohl die politischen Instanzen eine englische Ausgabe nie in Betracht zogen, wurde einmal in der Versammlung der wissenschaftlichen Beiräte des HLS, die anfangs jährlich tagte, der Ruf nach einer englischen Ausgabe des Werkes laut. Allerdings war nie ganz klar, wie ernsthaft der Vorschlag überhaupt gemeint und wie weit er durchdacht war. Man begegnet bisweilen der Ansicht, das Englische sei mit Vorzug für die sog. harten Wissenschaften anzuwenden, wo es vornehmlich um die präzise Übertragung von Informationen technischer Natur gehe. Für andere, geisteswissenschaftliche Disziplinen sei diese Sprache weniger geeignet, weil das Englische die Feinheiten und Nuancen eines Textes nur unzureichend wiedergeben könne. Auffällig ist bei solchen Äusserungen, dass das Englische zugleich auf- und abgewertet wird: aufgewertet, weil man davon ausgeht, dass sich diese Sprache für Wichtiges und Exaktes par excellence eignet; abgewertet hingegen, da man ihr die Fähigkeit, Zwischentöne auszudrücken, quasi abspricht.

Diese Einstellung dem Englischen gegenüber beruht gewiss zu einem guten Teil darauf, dass sie vor allem von Personen vertreten wird, die Englisch gerade nicht als Mutter- oder Hauptsprache sprechen. Meines Erachtens sind Überlegungen zur "Eignung" des Englischen für bestimmte Fächer gar nicht von Belang, sondern ganz praktische Aspekte, denn ein englisches HLS würde zahlreiche Fragen bezüglich Leserschaft und Arbeitsorganisation aufwerfen:

Hätte diese Ausgabe alle anderen ersetzt oder hätte sie diese ergänzt? An welches Publikum hätte sie sich gerichtet? An ausländische Leser oder an die Schweizer, von denen man annahm, dass sie bald einmal ausnahmslos Englisch beherrschen würden? In gewissen Kantonen scheinen die Verantwortlichen der Bildungspolitik tatsächlich davon auszugehen, dass in etwa 20 Jahren alle Schweizerinnen und Schweizer des Englischen so mächtig sind, dass dieses zur lingua franca sogar innerhalb der Eidgenossenschaft würde. ${ }^{10}$ Liesse sich dieses Ziel, dessen Nutzen und Machbarkeit Stoff für lange Diskussionen böte, wirklich erreichen, so würde Englisch bald einmal zum "Markenzeichen" einer bestimmten Generation bzw. zur Trennlinie von Generationen. Konkret auf ein englisches HLS bezogen, hiesse dies: Die Jungen könnten es problemlos konsultieren, während viele Ältere Verständnisschwierigkeiten hätten.

Das HLS visiert aber ein möglichst breites Publikum an, nicht nur Spezialisten der Geschichtswissenschaft, nicht nur Personen mit fundierter Sprachausbildung und nicht vornehmlich Junge, die Englisch als beste Fremdsprache beherrschen. Die Sprache sollte für keinen Schweizer ein Hindernis darstellen, das Lexikon zu konsultieren. Es wäre überdies sehr schwierig, ja absurd, die Geschichte eines Landes in einer Sprache auszudrücken, die dem vermittelten Inhalt völlig fremd ist, weil deren Begriffe nicht für die Realitäten der Schweiz und deren Bewohner geschaffen wurden. Spinnt man den hypothetischen Faden des

\footnotetext{
10 Die Schweiz ist diesbezüglich gespalten. Während die Ost- und Innerschweiz dem Englischen den Vorrang gibt und Appenzell Innerrhoden als erster Kanton 2001 Frühenglisch eingeführt hat, hält es Bern mit den Nordwest- und Westschweizer Kantonen, welche die Nachbarssprache als erste Fremdsprache bevorzugen.
} 
englischen HLS noch weiter, ergäben sich weitere handfeste Schwierigkeiten: Wer hätte in diesem Fall die Redaktionsarbeit übernommen? Etwa angelsächsische Historiker? Für die Übersetzung hätte sich ein besonderes, schier unlösbares Problem gestellt: Wo findet man englischsprachige Übersetzer, die gleichzeitig so mit der Schweizer Geschichte vertraut sind, dass sie auf diesem Gebiet arbeiten könnten?

Sobald solche praktischen Aspekte gründlich durchdacht werden, rückt die Vorstellung eines englischen HLS in weite Ferne. Auch wenn man nur ein Lexikon in einer einzigen Landessprache hätte erstellen wollen, so wäre die Arbeit ohne Übersetzung gar nicht möglich gewesen. Die Redaktoren suchen als Verfasser - unabhängig von deren Muttersprache - die besten Kenner eines Themas, ${ }^{11}$ und da die Spezialisten aus allen Teilen der Schweiz stammen, sind sie zwangsläufig nicht nur ein- und derselben Zunge. Nicht zu leugnen ist, dass ein einsprachiges HLS wohl schon nahe am Abschluss stünde, während die mehrsprachige Ausgabe noch ein paar weitere arbeitsintensive Jahre beanspruchen wird. Aber nicht zuletzt wegen der vollen Dreisprachigkeit ist das HLS bereits jetzt ein grosser kommerzieller Erfolg, ist doch gerade die italienische Ausgabe, erschienen in 1000 Exemplaren, bereits nahezu vergriffen.

$* * * * * *$

Die Gesamtausgaben des HLS haben sich im Jahr 2003 auf rund vier Millionen Franken belaufen, davon sind etwa 10\% für die Übersetzung der Artikel ausgegeben worden. Dieser Prozentsatz verändert sich pro Jahr nur geringfügig. So wird die Dreisprachigkeit der insgesamt zwölf Bände des Lexikons etwa fünf Millionen Franken erfordern. Fünf Millionen scheinen eine hohe Summe für eine Leistung, die nicht rein utilitaristische, sondern auch politische Zwecke verfolgt. Sie ist aber klein gemessen am immensen Arbeitsaufwand, der dahinter steckt, müssen doch pro Band 75'000 Zeilen aus den verschiedensten Gebieten der Geschichtswissenschaft übersetzt werden.

Wer weiterhin moniert, die Mehrsprachigkeit des HLS sei ein reines Zeichen politischer Korrektheit und verschlinge unnötige Summen, nehme immerhin eines zur Kenntnis: Nichts enthüllt Unklarheiten eines Textes erbarmungsloser als der genaue Vergleich von Original und Übersetzung. Die Hauptaufgabe des Redaktors, nämlich in Parallellektüre zu prüfen, ob sich der deutsche, französische und italienische Text inhaltlich entsprechen, fördert schonungslos Zusätze, Auslassungen und Fehler zutage. Die Übersetzung wird damit zur unschlagbaren Qualitätskontrolle des Gesamtwerks. Dank der Übersetzung pflegt das HLS die Landessprachen und damit auch das Bewusstsein, dass jeder Landesteil einen unverzichtbaren Bestandteil der schweizerischen Kultur darstellt. Deshalb ist die Erstellung eines Nachschlagewerkes in den vier Landessprachen nicht nur von sprachpolitischer, sondern auch von grösster kultureller Bedeutung für die Schweiz. Eine englische Ausgabe hätte diese Aufgaben nicht erfüllt und somit wahrhaft unnötige Mittel verschlungen.

\footnotetext{
${ }^{11}$ Der Artikel zu 'China' z.B. trägt die Unterschrift eines Tessiner Autors.
} 
Die Autorin ist diplomierte Übersetzerin (ETI, Genf) und hat ein Lizenziat in Geschichte und Deutsch.

Die Gesprächspartner:

- Pierre-G. Martin, Historiker, Übersetzer (seit 1993) und Redaktor (seit 1994) in der französischen Redaktion des HLS.

- Chiara Orelli, Historikerin, verantwortliche Redaktorin der italienischen Abteilung des HLS.

- Elmar Meier, diplomierter Übersetzer (DOZ, Zürich) und Terminologe, hat einige Semester Geschichte und Deutsch studiert.

\section{Literaturangaben}

Die Schweiz vom Paläolithikum bis zum frühen Mittelalter (La Suisse du Paléolithique à l'aube du Moyen Âge - La Svizzera del Paleolitico all'Alto Medio Evo) (1993). Ed. von der Schweizer Gesellschaft für Ur- und Frühgeschichte. Basel.

Drack, Walter/Fellmann, Rudolf (1988): Die Römer in der Schweiz. Stuttgart/Jona. (Drack, W./Fellmann R. (1992): La Suisse gallo-romaine. Lausanne.)

Drummer, Heike/Julien, Raymond/Loscertales, Javier/Stammerjohann, Harro (eds.) (1991): Französisch für Historiker. Fachsprachlicher Wortschatz mit Hinweisen zum Geschichtsstudium in Frankreich. Berlin.

Furrer, Norbert (1991): Glossarium Helvetiae historicorum I: Ortsnamen - toponymes - toponimi - nums locals. Bern.

Haberkern, Eugen/Wallach, Joseph F. (2001): Hilfswörterbuch für Historiker. 2 Bd., 9. Aufl. Tübingen. (= Uni-Taschenbücher 119).

Geographisches Lexikon der Schweiz (Dictionnaire géographique de la Suisse) (1902-1910). 6 Bd. Neuenburg.

Historisch-biographisches Lexikon der Schweiz (Dictionnaire historique et biographique de la Suisse) (1921-1934). 7 Bd. Neuenburg.

Historisches Lexikon der Schweiz. Ed. von der Stiftung Historisches Lexikon der Schweiz (HLS). Basel. (Dictionnaire historique de la Suisse. Publié par la Fondation Dictionnaire historique de la Suisse (DHS). Hauterive (NE). - Dizionario storico della Svizzera. A cura della Fondazione Dizionario storico della Svizzera (DSS). Locarno.)

Martin, Pierre-G. (2001): "Histoire, dictionnaire et traduction : Le Dictionnaire historique de la Suisse". Hieronymus. Vierteljahresschrift für Übersetzungspraxis der ASTTI (Schweizerischer Übersetzer- und Dolmetscherverband) 3: S. 23-27.

Vischer, Lukas (1998): Ökumenische Kirchengeschichte der Schweiz. 2. korr. Aufl. Freiburg/Basel. (Vischer, L. (1994): Histoire du Christianisme en Suisse. Genève/Fribourg.) 